\title{
Influence of phonon renormalization in Eliashberg theory for superconductivity in two- and three-dimensional systems
}

\author{
Fabian Schrodi $\odot,{ }^{*}$ Alex Aperis $\odot,{ }^{\dagger}$ and Peter M. Oppeneer $\odot^{*}$ \\ Department of Physics and Astronomy, Uppsala University, P.O. Box 516, SE-75120 Uppsala, Sweden
}

(Received 13 October 2020; revised 4 February 2021; accepted 15 February 2021; published 24 February 2021)

\begin{abstract}
Eliashberg's foundational theory of superconductivity is based on the application of Migdal's approximation, which states that vertex corrections to lowest-order electron-phonon scattering are negligible if the ratio between phonon and electron energy scales is small. The resulting theory incorporates the first Feynman diagrams for electron and phonon self-energies. However, the latter is most commonly neglected in numerical analyses. Here we provide an extensive study of full-bandwidth Eliashberg theory in two and three dimensions, where we include the full back reaction of electrons onto the phonon spectrum. We unravel the complex interplay between nesting properties, size of the Fermi surface, renormalized electron-phonon coupling, phonon softening, and superconductivity. We propose furthermore a scaling law for the maximally possible critical temperature $T_{c}^{\max } \propto \lambda(\Omega) \sqrt{\Omega_{0}^{2}-\Omega^{2}}$ in two- and three-dimensional systems, which embodies both the renormalized electronphonon coupling strength $\lambda(\Omega)$ and softened phonon spectrum $\Omega$. Also, we analyze for which electronic structure properties a maximal $T_{c}$ enhancement can be achieved.
\end{abstract}

DOI: 10.1103/PhysRevB.103.064511

\section{INTRODUCTION}

The current state-of-the-art description of superconductors is Eliashberg theory [1], which is especially applied in cases where the more simplified BCS (Bardeen-Cooper-Schrieffer) treatment [2] cannot capture the main characteristics of a given system. One of the key aspects to the success of Eliashberg theory is the applicability of Migdal's approximation [3], which states that higher-order Feynman diagrams for electronphonon scattering can be neglected if the ratio of phonon to electron energy scale is a small number. In such a case it is sufficient to only consider all lowest-order Feynman diagrams for the electron and phonon self-energy, although the latter is neglected in most cases. Such a neglect may be motivated by the drive for making an extremely complicated problem easier. Interestingly though, in the original works by Migdal [3] and Eliashberg [1] the phonon self-energy was included in the calculation in an approximative way. However, for a quantitative analysis it is a generally accepted procedure to neglect the back reaction of electrons onto the phonon spectrum.

In available literature the phonon renormalization is most commonly considered only when checking the validity of Eliashberg theory calculations [4-7]. The numerical results are then benchmarked against outcomes of quantum Monte

\footnotetext{
*fabian.schrodi@physics.uu.se

†alex.aperis@physics.uu.se

‡peter.oppeneer@physics.uu.se
}

Published by the American Physical Society under the terms of the Creative Commons Attribution 4.0 International license. Further distribution of this work must maintain attribution to the author(s) and the published article's title, journal citation, and DOI. Funded by Bibsam.
Carlo (QMC) [8,9] or dynamical mean-field theory simulations [10]. Comparing conclusions from various authors does not necessarily lead to a completely coherent picture concerning the validity of Eliashberg theory, but there is consensus about the existence of a maximal electron-phonon coupling strength marking the border of applicability. Characteristics of the superconducting state in such studies are found only by extrapolation from normal-state properties, and, in addition, the QMC calculations are performed on relatively small lattices due to the huge complexity of the problem. The most commonly studied system in these works is the two-dimensional (2D) Holstein model [8,11-16], often with additional constraints such as half filling, while three-dimensional (3D) systems are rarely considered in numerical calculations, presumably due to the large computational complexity.

Another way of checking the validity of the commonly employed Migdal approximation, and hence of the resulting Eliashberg theory, is to compute vertex corrections corresponding to additional Feynman diagrams. This has been attempted in various works under different kinds of approximation [17-26]. The first vertex-corrected self-consistent Eliashberg theory without further simplifications has been recently proposed by the current authors [27]. All these works have in common that one or more additional Feynman diagrams to the electron-phonon interaction are studied and compared to the commonly employed Eliashberg formalism, that does not include a finite phonon self-energy.

The aim of our current paper is to give a comprehensive overview of the influence of phonon renormalization occurring within Eliashberg theory when the lowest-order Feynman diagram for the phonon self-energy is taken self-consistently into account, rather than commenting much on its validity with respect to other theories. Our efficient implementation not only allows one to access sufficiently low temperatures to study the superconducting state without relying on 
extrapolation of normal-state properties, but also opens the discussion of 3D systems, which has so far been elusive in available literature. Our theory is based on a Holstein-like Hamiltonian, containing a nearest-neighbor tight-binding model for the electron energies, an isotropic Einstein phonon mode, and isotropic electron-phonon scattering elements, controlling the coupling strength in the system. We explore here in detail the interplay of phonon softening, renormalized coupling strength, nesting properties, superconducting energy gap, and, eventually, the maximally possible transition temperature $T_{c}$.

Our theory takes the bare phonon frequency $\Omega_{0}$, coupling strength $\lambda_{0}$, and electron energies $\xi_{\mathbf{k}}$ as inputs. By varying these quantities we pinpoint the renormalized coupling strength and phonon softening (momentum dependent decrease in magnitude) as key ingredients to how our selfconsistent results change. Other crucially important aspects for the interacting state are Fermi-surface (FS) nesting properties of $\xi_{\mathbf{k}}$ and the size of the FS. There exists a critical value $\lambda_{0}^{\star}$ of the bare input coupling, at which the phonon energies become negative, indicating a lattice instability. Another important value of $\lambda_{0}$ marks the onset of superconductivity, $\lambda_{0}^{\Delta}$. In two dimensions we find an enhancement of both $\lambda_{0}^{\star}$ and $\lambda_{0}^{\Delta}$ with increased shallowness of $\xi_{\mathbf{k}}$, which goes along with less coherent nesting properties and a decrease in FS size. Notably, we find that our model systems exhibit maximal superconducting transition temperatures $T_{c}$ for an intermediate system that is not very shallow, but also not ideally nested. For 3D systems the significance of FS nesting is weakened, such that trends in $\lambda_{0}^{\star}, \lambda_{0}^{\Delta}$, and maximum $T_{c}$ are mainly dictated by the FS size. An exception to these tendencies is our most shallow $\xi_{\mathbf{k}}$ considered in three dimensions, because in this particular system nesting is exceptionally coherent due to the special role taken by the $\Gamma$ point of the Brillouin zone (BZ).

From here we proceed as follows: In Sec. II we introduce the formalism and mathematical steps needed for deriving a self-consistent and full-bandwidth Eliashberg theory, that includes all lowest-order processes for electron and phonon self-energies. The terminology "full bandwidth" is used here to stress that our equations are solved numerically by taking into account the complete bandwidth of electron energies $\xi_{\mathbf{k}}$, rather than focusing on a narrow energy window around the Fermi level, as is common practice in Fermi-surface restricted calculations. We provide some benchmark checks of our implementation in the Appendix. We continue by introducing electron dispersions in two and three dimensions (see Sec. III). In particular, we define in both cases three different energies via varying the chemical potential, that differ in nesting and FS properties. In Sec. IV follows an exploration of phase space, spanned by the different input parameters to our theory. We discuss in detail the aspects of phonon softening, renormalization of electron-phonon coupling, and the onset of superconductivity, and try to unravel their complex interplay by deriving approximate relations between them. In Sec. V the subject is a closer discussion of the superconducting energy gap as function of temperature, and consequently the transition temperatures, in two and three dimensions. Motivated by the precedent findings we propose a scaling law for the maximum transition temperature that models our numerical results to very good accuracy. The paper is concluded in Sec. VI with a brief discussion on related works, possible extensions to our theory, and potential future directions.

\section{THEORY}

We consider a phonon mode with frequency $\Omega_{0}$ and electron-phonon coupling that is given by $g_{\mathbf{q}}$. Here the electronic energies are modeled by a single band dispersion $\xi_{\mathbf{k}}$, with $\mathbf{k}$ a BZ wave vector. By setting $\mathbf{q}=\mathbf{k}-\mathbf{k}^{\prime}$ we can write the Hamiltonian as

$$
\begin{aligned}
H= & \sum_{\mathbf{k}} \xi_{\mathbf{k}} \Psi_{\mathbf{k}}^{\dagger} \hat{\rho}_{3} \Psi_{\mathbf{k}}+\sum_{\mathbf{q}} \hbar \Omega_{0}\left(b_{\mathbf{q}}^{\dagger} b_{\mathbf{q}}+\frac{1}{2}\right) \\
& +\sum_{\mathbf{k}, \mathbf{k}^{\prime}} g_{\mathbf{k}-\mathbf{k}^{\prime}} u_{\mathbf{k}-\mathbf{k}^{\prime}} \Psi_{\mathbf{k}^{\prime}}^{\dagger} \hat{\rho}_{3} \Psi_{\mathbf{k}} .
\end{aligned}
$$

Above we use $b_{\mathbf{q}}^{\dagger}$ and $b_{\mathbf{q}}$ as bosonic creation and annihilation operators, which determine the phonon displacement $u_{\mathbf{q}}=$ $b_{\mathbf{q}}+b_{-\mathbf{q}}^{\dagger}$. The electronic creation and annihilation operators $c_{\mathbf{k}, \sigma}^{\dagger}$ and $c_{\mathbf{k}, \sigma}$ are part of the Nambu spinor $\Psi_{\mathbf{k}}^{\dagger}=\left(c_{\mathbf{k}, \uparrow}^{\dagger}, c_{-\mathbf{k}, \downarrow}\right)$, where $\sigma \in\{\uparrow, \downarrow\}$ is a spin label. The imaginary time $\tau$ dependent electron and phonon Green's functions are, respectively, given by

$$
\begin{gathered}
\hat{G}_{\mathbf{k}}(\tau)=-\left\langle\mathcal{T}_{\tau} \Psi_{\mathbf{k}}(\tau) \otimes \Psi_{\mathbf{k}}^{\dagger}(0)\right\rangle, \\
D_{\mathbf{q}}(\tau)=-\left\langle\mathcal{T}_{\tau} u_{\mathbf{q}}(\tau) u_{\mathbf{q}}(0)\right\rangle,
\end{gathered}
$$

where $\mathcal{T}_{\tau}$ is the imaginary-time ordering operator. Both propagators obey a Dyson equation in Matsubara space, reading

$$
\begin{gathered}
\hat{G}_{\mathbf{k}, m}=\hat{G}_{\mathbf{k}, m}^{0}+\hat{G}_{\mathbf{k}, m}^{0} \hat{\Sigma}_{\mathbf{k}, m} \hat{G}_{\mathbf{k}, m}, \\
D_{\mathbf{q}, l}=D_{\mathbf{q}, l}^{0}+D_{\mathbf{q}, l}^{0} \Pi_{\mathbf{q}, l} D_{\mathbf{q}, l},
\end{gathered}
$$

where we make use of the notation $f\left(\mathbf{k}, i \omega_{m}\right)=f_{\mathbf{k}, m}$ with Matsubara frequencies $\omega_{m}=\pi T(2 m+1)$ for generic fermion functions $f$, and $h\left(\mathbf{q}, i q_{l}\right)=h_{\mathbf{q}, l}$ for boson functions $h$, with $q_{l}=2 \pi T l$. The above Eqs. (4) and (5) are solved for the dressed propagators as functions of the respective self-energies $\hat{\Sigma}_{\mathbf{k}, m}$ and $\Pi_{\mathbf{q}, l}$ :

$$
\begin{aligned}
\hat{G}_{\mathbf{k}, m}^{-1} & =\left[\hat{G}_{\mathbf{k}, m}^{0}\right]^{-1}-\hat{\Sigma}_{\mathbf{k}, m}, \\
D_{\mathbf{q}, l}^{-1} & =\left[D_{\mathbf{q}, l}^{0}\right]^{-1}-\Pi_{\mathbf{q}, l} .
\end{aligned}
$$

The noninteracting Green's functions are defined by

$$
\begin{gathered}
{\left[\hat{G}_{\mathbf{k}, m}^{0}\right]^{-1}=i \omega_{m} \hat{\rho}_{0}-\xi_{\mathbf{k}} \hat{\rho}_{3}} \\
{\left[D_{\mathbf{q}, l}^{0}\right]^{-1}=\left(\frac{1}{i q_{l}-\Omega_{0}}-\frac{1}{i q_{l}+\Omega_{0}}\right)^{-1}=-\frac{1}{2 \Omega_{0}}\left(q_{l}^{2}+\Omega_{0}^{2}\right),}
\end{gathered}
$$

where we use the Pauli matrices $\hat{\rho}_{i}, i \in\{0,1,2,3\}$. The electronic self-energy can be decomposed to the mass enhancement function $Z_{\mathbf{k}, m}$, chemical potential renormalization $\Gamma_{\mathbf{k}, m}$, and superconducting order parameter $\phi_{\mathbf{k}, m}$ :

$$
\hat{\Sigma}_{\mathbf{k}, m}=i \omega_{m}\left(1-Z_{\mathbf{k}, m}\right) \hat{\rho}_{0}+\Gamma_{\mathbf{k}, m} \hat{\rho}_{3}+\phi_{\mathbf{k}, m} \hat{\rho}_{1} .
$$


(a)

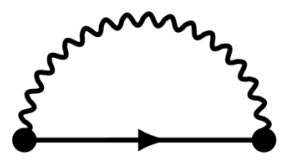

(b)

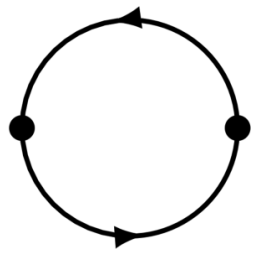

FIG. 1. (a, b) Lowest-order Feynman diagram for the electron and phonon self-energy, respectively.

Together with Eq. (6) this leads to an inverse electron Green's function

$$
\hat{G}_{\mathbf{k}, m}^{-1}=i \omega_{m} Z_{\mathbf{k}, m} \hat{\rho}_{0}-\left(\xi_{\mathbf{k}}+\Gamma_{\mathbf{k}, m}\right) \hat{\rho}_{3}-\phi_{\mathbf{k}, m} \hat{\rho}_{1},
$$

so that after matrix inversion we get

$$
\begin{gathered}
\hat{G}_{\mathbf{k}, m}=\left[i \omega_{m} Z_{\mathbf{k}, m} \hat{\rho}_{0}+\left(\xi_{\mathbf{k}}+\Gamma_{\mathbf{k}, m}\right) \hat{\rho}_{3}+\phi_{\mathbf{k}, m} \hat{\rho}_{1}\right] \Theta_{\mathbf{k}, m}^{-1}, \\
\Theta_{\mathbf{k}, m}=\left(i \omega_{m} Z_{\mathbf{k}, m}\right)^{2}-\left(\xi_{\mathbf{k}}+\Gamma_{\mathbf{k}, m}\right)^{2}-\phi_{\mathbf{k}, m}^{2}
\end{gathered}
$$

In this paper we take into account all lowest-order processes in both the electron and phonon self-energies. The corresponding Feynman diagrams are shown in Figs. 1(a) and 1(b). By exploiting momentum and energy conservation in the scattering processes we can translate the Feynman diagram for the electron self-energy into

$$
\hat{\Sigma}_{\mathbf{k}, m}=-T \sum_{\mathbf{k}^{\prime}, m^{\prime}}\left|g_{\mathbf{k}-\mathbf{k}^{\prime}}\right|^{2} D_{\mathbf{k}-\mathbf{k}^{\prime}, m-m^{\prime}} \hat{\rho}_{3} \hat{G}_{\mathbf{k}^{\prime}, m^{\prime}} \hat{\rho}_{3},
$$

and for the phonon self-energy we obtain

$$
\Pi_{\mathbf{q}, l}=T\left|g_{\mathbf{q}}\right|^{2} \sum_{\mathbf{k}, m} \operatorname{Tr}\left\{\hat{\rho}_{3} \hat{G}_{\mathbf{k}, m} \hat{\rho}_{3} \hat{G}_{\mathbf{k}+\mathbf{q}, m+l}\right\} .
$$

At this point it is convenient to define the electron-phonon interaction kernel

$$
V_{\mathbf{q}, l}=-\left|g_{\mathbf{q}}\right|^{2} D_{\mathbf{q}, l},
$$

so that we can write Eq. (14) as

$$
\hat{\Sigma}_{\mathbf{k}, m}=T \sum_{\mathbf{k}^{\prime}, m^{\prime}} V_{\mathbf{k}-\mathbf{k}^{\prime}, m-m^{\prime}} \hat{\rho}_{3} \hat{G}_{\mathbf{k}^{\prime}, m^{\prime}} \hat{\rho}_{3} .
$$

Combining Eq. (17) with Eqs. (10) and (12) leads to the Eliashberg equations

$$
\begin{gathered}
Z_{\mathbf{k}, m}=1-\frac{T}{\omega_{m}} \sum_{\mathbf{k}^{\prime}, m^{\prime}} V_{\mathbf{k}-\mathbf{k}^{\prime}, m-m^{\prime}} \frac{\omega_{m^{\prime}} Z_{\mathbf{k}^{\prime}, m^{\prime}}}{\Theta_{\mathbf{k}^{\prime}, m^{\prime}}}, \\
\Gamma_{\mathbf{k}, m}=T \sum_{\mathbf{k}^{\prime}, m^{\prime}} V_{\mathbf{k}-\mathbf{k}^{\prime}, m-m^{\prime}} \frac{\xi_{\mathbf{k}^{\prime}}+\Gamma_{\mathbf{k}^{\prime}, m^{\prime}}}{\Theta_{\mathbf{k}^{\prime}, m^{\prime}}}, \\
\phi_{\mathbf{k}, m}=-T \sum_{\mathbf{k}^{\prime}, m^{\prime}} V_{\mathbf{k}-\mathbf{k}^{\prime}, m-m^{\prime}} \frac{\phi_{\mathbf{k}^{\prime}, m^{\prime}}}{\Theta_{\mathbf{k}^{\prime}, m^{\prime}}},
\end{gathered}
$$

from which the superconducting gap function can be defined as $\Delta_{\mathbf{k}, m}=\phi_{\mathbf{k}, m} / Z_{\mathbf{k}, m}$. Inserting Eq. (12) into Eq. (15) gives

the phonon self-energy as function of $Z_{\mathbf{k}, m}, \Gamma_{\mathbf{k}, m}$, and $\phi_{\mathbf{k}, m}$ :

$$
\begin{aligned}
\Pi_{\mathbf{q}, l}= & -2 T\left|g_{\mathbf{q}}\right|^{2} \sum_{\mathbf{k}, m}\left(\frac{\omega_{m} Z_{\mathbf{k}, m}}{\Theta_{\mathbf{k}, m}} \frac{\omega_{m+l} Z_{\mathbf{k}+\mathbf{q}, m+l}}{\Theta_{\mathbf{k}+\mathbf{q}, m+l}}\right. \\
& \left.-\frac{\xi_{\mathbf{k}}+\Gamma_{\mathbf{k}, m}}{\Theta_{\mathbf{k}, m}} \frac{\xi_{\mathbf{k}+\mathbf{q}}+\Gamma_{\mathbf{k}+\mathbf{q}, m+l}}{\Theta_{\mathbf{k}+\mathbf{q}, m+l}}+\frac{\phi_{\mathbf{k}, m}}{\Theta_{\mathbf{k}, m}} \frac{\phi_{\mathbf{k}+\mathbf{q}, m+l}}{\Theta_{\mathbf{k}+\mathbf{q}, m+l}}\right) .
\end{aligned}
$$

The above equations are solved self-consistently in an iterative manner: Assuming $Z_{\mathbf{k}, m}, \Gamma_{\mathbf{k}, m}$, and $\phi_{\mathbf{k}, m}$ are known from a previous iteration, we first calculate the phonon selfenergy via Eq. (21), by using also Eq. (13). With $\Pi_{\mathbf{q}, l}$ at hand, and the bare phonon propagator in Eq. (9), we calculate the phonon Green's function from Eq. (7), which then determines the electron-phonon interaction kernel Eq. (16). As final step we solve for the mass renormalization, the chemical potential renormalization, and the gap function via Eqs. (18)-(20). This process is repeated until convergence is reached. From the results we can calculate the electron filling as

$$
n=1-2 T \sum_{\mathbf{k}, m} \frac{\xi_{\mathbf{k}}+\Gamma_{\mathbf{k}, m}}{\Theta_{\mathbf{k}, m}} .
$$

The input to our theory is the electron dispersion $\xi_{\mathbf{k}}$, the phonon frequency $\Omega_{0}$, and the coupling $\lambda_{0}$, which can be expressed as

$$
\lambda_{0}=\left\langle\left\langle\lambda_{\mathbf{k}-\mathbf{k}^{\prime}}\right\rangle_{\mathbf{k} \in \mathrm{FS}}\right\rangle_{\mathbf{k}^{\prime} \in \mathrm{FS}}=\frac{2 N_{0}}{\Omega_{0}}\left\langle\left\langle\left|g_{\mathbf{k}-\mathbf{k}^{\prime}}\right|^{2}\right\rangle_{\mathbf{k} \in \mathrm{FS}}\right\rangle_{\mathbf{k}^{\prime} \in \mathrm{FS}},
$$

with $N_{0}$ the density of states (DOS) at the FS. After solving self-consistently for the interacting state we have access to the renormalized coupling strength $\lambda_{\mathbf{q}}$ and frequencies $\Omega_{\mathbf{q}}$. These are, respectively, given as

$$
\begin{gathered}
\lambda_{\mathbf{q}}=-N_{0}\left|g_{\mathbf{q}}\right|^{2} D_{\mathbf{q}, l=0}, \\
\Omega_{\mathbf{q}}=\sqrt{\Omega_{0}^{2}+2 \Omega_{0} \Pi_{\mathbf{q}, l=0},}
\end{gathered}
$$

and it is convenient to define $\lambda=\left\langle\left\langle\lambda_{\mathbf{k}-\mathbf{k}^{\prime}}\right\rangle_{\mathbf{k} \in \mathrm{FS}}\right\rangle_{\mathbf{k}^{\prime} \in \mathrm{FS}}$ as a measure of the total coupling strength in the system. A nonvanishing imaginary part of $\Omega_{\mathbf{q}}$ marks a lattice instability.

The theory presented here has been included in the UPPSALA SUPERCONDUCTIVITY code (UppSC) [28-33], and we benchmark our implementation in the Appendix against existing literature [8]. Momentum and frequency summations are carried out via efficient Fourier convolution techniques. For calculations in two dimensions (three dimensions) we use $32 \times 32(32 \times 32 \times 32)$ points for the $\mathbf{k}$ and $\mathbf{q}$ grids. The number of Matsubara frequencies is always chosen larger than 2000. The equations given in our current paper are formulated and solved in Matsubara space. Analogously, one can build up a theory as function of real frequencies, as has been done in a recent work by Nosarzewski et al., where the authors discuss the impact of phonon softening on various spectral properties [34].

\section{MODEL SYSTEMS}

In this section we introduce two kinds of electron dispersions that are employed in the rest of our paper. Starting in 

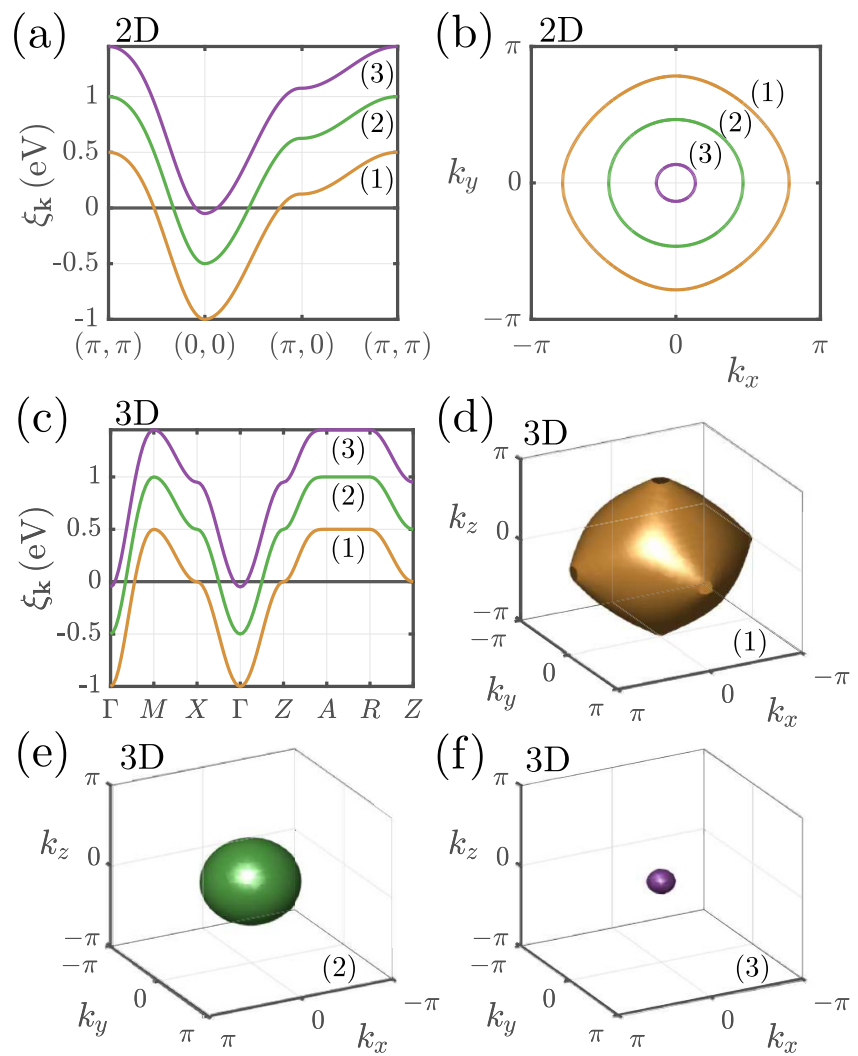

FIG. 2. (a) Two-dimensional electron energies along highsymmetry lines of the Brillouin zone. The purple, green, and orange curves are found from Eq. (26) by choosing $\mu=-887.5,-437.5$, and $62.5 \mathrm{meV}$. (b) Fermi surfaces corresponding to panel (a), drawn in similar color code. (c) Three-dimensional electron dispersion $\xi_{\mathbf{k}}$ as calculated from Eq. (27) for different chemical potentials (orange, $\mu=-0.125 \mathrm{eV}$; green, $\mu=-0.625 \mathrm{eV}$; purple, $\mu=-1.075 \mathrm{eV}$ ), shown along high-symmetry lines. (d-f) Three-dimensional Fermi surfaces colored in correspondence to panel (c). In both two and three dimensions we use labels (1), (2), and (3) to refer to the electron energies depicted by orange, green, and purple colors, respectively.

two spatial dimensions we define the electron energies as

$$
\begin{aligned}
\xi_{\mathbf{k}}= & -2 t^{(1)}\left[\cos \left(k_{x}\right)+\cos \left(k_{y}\right)\right] \\
& -4 t^{(2)} \cos \left(k_{x}\right) \cos \left(k_{y}\right)-\mu,
\end{aligned}
$$

where $t^{(1)}$ and $t^{(2)}$ are the nearest- and next-nearest-neighbor hopping energies of our tight-binding model, and $\mu$ is the chemical potential. For simplicity we assume here a tetragonal square-lattice structure in two dimensions, but our results hold qualitatively also for different symmetries. Whenever referring to a $2 \mathrm{D}$ system, we choose $t^{(1)}=W / 8$ and $t^{(2)}=$ $t^{(1)} / 4$, where $W=1.5 \mathrm{eV}$ is the electronic bandwidth. We test three different examples for the choice of $\mu$, the resulting dispersions along high-symmetry lines of the $\mathrm{BZ}$ are shown in Fig. 2(a), while corresponding Fermi surfaces (same color code) are plotted in Fig. 2(b). The orange (1), green (2), and purple (3) curves are, respectively, found using $\mu=62.5$, -437.5 , and $-887.5 \mathrm{meV}$, and correspond to high, moderate, and low electron fillings.
Turning now to the case of three spatial dimensions, we use the electron energies

$$
\begin{aligned}
\xi_{\mathbf{k}}= & -2 t^{(1)} \sum_{i=x, y, z} \cos \left(k_{i}\right) \\
& -2 t^{(2)} \sum_{i=x, y, z} \prod_{j=x, y, z ; j \neq i} \cos \left(k_{j}\right)-\mu .
\end{aligned}
$$

Similarly as before we use an electronic bandwidth $W=$ $1.5 \mathrm{eV}$ and fix the hopping energies as $t^{(1)}=W / 8, t^{(2)}=$ $t^{(1)} / 4$. We define three dispersions that are shown in Fig. 2(c) along high-symmetry lines of the simple cubic BZ. The orange (1), green (2), and purple (3) lines represent the choices $\mu=-0.125,-0.625$, and $-1.075 \mathrm{eV}$, respectively. Again, the resulting electron fillings are high, moderate, and low. The FS shown in Fig. 2(d) is very large and resembles to a good approximation an ideal Fermi gas. As the opposite case, we have an extremely shallow energy dispersion reflected by the tiny FS in Fig. 2(f). In Fig. 2(e) we show an intermediate case. To avoid any possible misunderstandings, we note that shallow bands are not to be confused with "flat bands" as the latter host electrons with vanishing velocities and therefore high electron DOS.

In the following we examine how the choice of electron dispersion affects our self-consistent results of the Eliashberg equations in two and three spatial dimensions. For simplification we consider here isotropic electron-phonon scattering, $g_{\mathbf{q}}=g_{0}$. As stated in Sec. II, the input to our Eliashberg equations is the bare electron-phonon coupling $\lambda_{0}$, the electron dispersion $\xi_{\mathbf{k}}$, and the bare phonon frequency $\Omega_{0}$. Since we want to study trends with respect to those input parameters we need to pay special attention to the electron filling that is calculated as function of the self-consistent results [see Eq. (22)]. To be able to compare outcomes for different $\Omega_{0}$, $\lambda_{0}$, and $T$ we need to ensure that $n$ stays constant, which we achieve by introducing an additional chemical potential shift $\delta \mu$, such that the input to the Eliashberg equations is $\xi_{\mathbf{k}}-\delta \mu[35]$.

\section{PHONON SOFTENING, COUPLING, AND SUPERCONDUCTING GAP}

As a first step we want to understand how the input parameters influence our self-consistent results. For this purpose we solve the Eliashberg equations in two dimensions as function of $\lambda_{0}$ at $T=20 \mathrm{~K}$ for various bare frequencies $\Omega_{0}$. We show our results for dispersions (1), (2), and (3) of Fig. 2(a), respectively, in the first, second, and third column of Fig. 3 in the colors representing each $\xi_{\mathbf{k}}$. In all of these columns we show results for different initial frequencies $\Omega_{0}$ with line styles as indicated in the legend of Fig. $3(\mathrm{~g})$. The upper row shows the renormalized coupling strength as function of $\lambda_{0}$, where we add as guide for the eye the case $\lambda=\lambda_{0}$ as solid gray line. The middle row shows the minimum renormalized phonon frequency, again as function of bare input coupling. The maximum superconducting gap, defined by $\Delta=\max _{\mathbf{k} \in \mathrm{BZ}} \Delta_{\mathbf{k}, m=0}$, is shown in the lower row.

In Figs. 3(a)-3(c) we observe the well-known behavior of the renormalized coupling with increasing $\lambda_{0}[5,8,10]$, i.e., $\lambda$ approaches a divergence, which we define to occur 

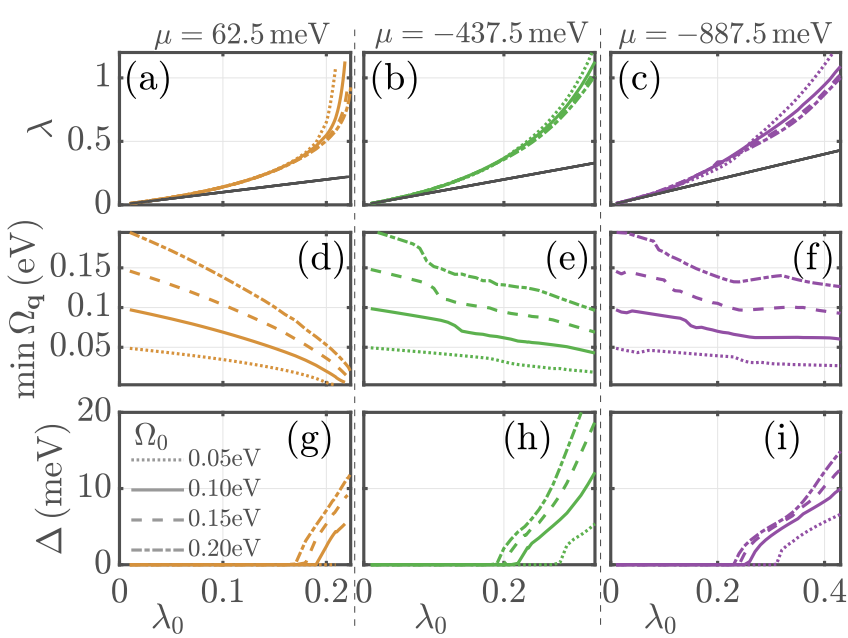

FIG. 3. Results for the renormalized coupling strength $\lambda$ (upper row), minimum renormalized phonon frequency (middle row), and maximum superconducting gap (lower row), calculated for our 2D systems. Results shown in the first, second, and third column are computed with the orange, green, and purple dispersion of Fig. 2(a), respectively. Different line styles correspond to bare phonon frequencies $\Omega_{0}$ as written in the legend of panel (g). The solid gray lines in panels (a) - (c) refer to $\lambda=\lambda_{0}$.

at $\lambda_{0}^{\star}$. As is apparent in Figs. 3(d)-3(f), when $\lambda_{0} \rightarrow \lambda_{0}^{\star}$ the minimal phonon frequency vanishes, indicating a lattice instability. The average frequency on the other hand decreases approximately linearly with $\lambda_{0}$ (not shown). Such tendencies towards a charge density wave instability occur at exchange momenta following the most coherent nesting wave vector for the respective electron dispersion (see discussion below and Fig. 4). The slight increase in minimum phonon frequency, seen for $\Omega_{0}=200 \mathrm{meV}$ in Fig. 3(f), can be interpreted as signature of a very nonadiabatic parameter choice, hence we can expect that this feature disappears upon the inclusion of vertex corrections. From Fig. 2(a) we know that the FS nesting condition is met less accurately as we go from the orange (1) to the purple dispersion (3). The phonon self-energy exhibits less coherent contributions for the shallow energy band (3), which in turn renormalizes the phonon propagator to a smaller extent. For this reason we find increasing values of $\lambda_{0}^{\star}$ as we go from the first to the third column in Fig. 3. In line with this observation we find the fastest decrease of minimum phonon frequencies as function of $\lambda_{0}$ in Fig. 3(d), and the slowest in Fig. 3(f).

From each of Figs. 3(g)-3(i) we observe that the superconducting gap opening with respect to $\lambda_{0}$ depends on $\Omega_{0}$. An increase in the initial phonon frequency reduces the minimal bare coupling strength needed for a finite superconducting gap. Further, comparing these three panels shows that the onset of superconductivity depends also on the electronic dispersion. This observation can again be understood in terms of changed nesting conditions (see the discussion above). When considering the superconducting gap as function of $\lambda$ (instead of $\lambda_{0}$ ), the difference in the onset of $\Delta \neq 0$ among results calculated for our three $2 \mathrm{D}$ electron dispersions becomes smaller. The onset of superconductivity with respect to $\lambda_{0}$ is discussed in more detail later in this section, and further analysis of the
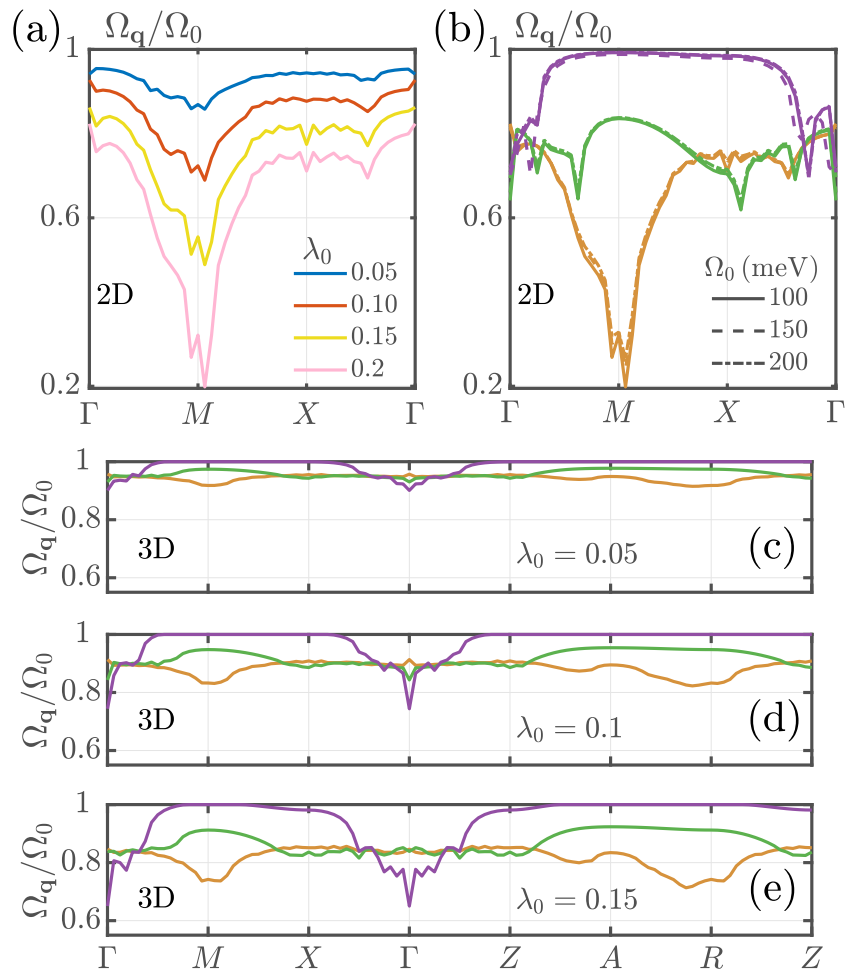

FIG. 4. Momentum dependent phonon frequencies, normalized to the initial choice of $\Omega_{0}$. Calculations were performed at $T=$ $20 \mathrm{~K}$. (a) Results for different bare couplings $\lambda_{0}$ as written in the legend, computed for $\Omega_{0}=100 \mathrm{meV}$ and the orange dispersion (1) of Fig. 2(a). (b) Different colors correspond to the choice of 2D electron dispersion [compare Fig. 2(a)]. With $\lambda_{0}=0.2$, the three different line styles represent varying choices of bare frequency $\Omega_{0}$ as written in the legend. (c-e) Renormalized phonon frequencies along high-symmetry lines of the BZ. Different colors correspond to 3D electron energies as shown in Fig. 2(c). Bare couplings are chosen as $\lambda_{0}=0.05,0.1$, and 0.15 .

superconducting state in connection with phonon renormalization is provided in Sec. V.

Including the back reaction of electrons on the phonon spectrum, via the Feynman diagram shown in Fig. 1(b), leads to a decrease in the magnitude of frequencies, which is a well-known behavior commonly referred to as phonon softening [36]. This phenomenon has been discussed especially in context of the 2D Holstein model $[4,5,12,15]$, and it marks tendencies of the system to develop a charge density wave instability. The question of whether phonon softening is favorable for superconductivity or suppresses $T_{c}$ is discussed in further detail in Sec. V.

To first show the general effect, let us consider the orange dispersion (1) in Fig. 2(a) and a bare frequency $\Omega_{0}=$ $100 \mathrm{meV}$. After self-consistently solving the Eliashberg equations in two dimensions at $T=20 \mathrm{~K}$, we calculate the renormalized phonon spectrum via Eq. (25). In Fig. 4(a) we plot our result for $\Omega_{\mathbf{q}} / \Omega_{0}$ along high-symmetry lines of the BZ for various coupling strengths as indicated in the legend. In the limit of small $\lambda_{0}$ (see blue curve), the renormalization effects are relatively minor, i.e., $\Omega_{\mathbf{q}} / \Omega_{0}$ stays close to unity throughout the BZ. The biggest phonon softening occurs at 
$\mathbf{q} \simeq(\pi, \pi)$ because the FS is relatively well nested at this exchange momentum. As we increase $\lambda_{0}$ we confirm the leading instability to be at $(\pi, \pi)$, since the smallest ratio of renormalized to bare frequencies is observed at this $\mathbf{q}$ [see Fig. 4(a)]. Additionally, phonon softening occurs throughout the whole BZ, so we observe $\Omega_{\mathbf{q}}<\Omega_{0} \forall \mathbf{q}$ for any finite $\lambda_{0}$.

We can understand the observed decreases in $\Omega_{\mathbf{q}}$ by expressing the phonon self-energy as $\Pi_{\mathbf{q}, l}=-g_{\mathbf{q}}^{2} \chi_{\mathbf{q}, l}^{0}$, where $\chi_{\mathbf{q}, l}^{0}$ is the charge susceptibility [15]. Inserting into Eq. (25) and using $g_{\mathbf{q}}=g_{0}$ gives $\Omega_{\mathbf{q}} / \Omega_{0}=$ $\sqrt{\Omega_{0}^{2}-2 \Omega_{0} g_{0}^{2} \chi_{\mathbf{q}, l=0}^{0}} / \Omega_{0}=\sqrt{1-2 g_{0}^{2} \chi_{\mathbf{q}, l=0}^{0} / \Omega_{0}}$. Therefore the anisotropy in the renormalized frequencies comes solely from the susceptibility, which in turn is dominated by contributions due to FS nesting. In addition we note that $g_{0}^{2} / \Omega_{0} \propto \lambda_{0}$, therefore the phonon softening is expected to increase with coupling strength, a trend confirmed by our calculations shown in Fig. 4(a).

To further examine the influence of nesting on the phonon renormalization we test the three dispersions as shown in Fig. 2(a). For each $\xi_{\mathbf{k}}$ we choose initial frequencies $\Omega_{0}=$ 100,150 , or $200 \mathrm{meV}$ and a coupling strength of $\lambda_{0}=0.2$. Our results for $\Omega_{\mathbf{q}} / \Omega_{0}$ are plotted in Fig. 4(b), again as function of exchange momentum $\mathbf{q}$ along high-symmetry lines. The color code is identical to Fig. 2(a), and we use varying line styles, as written in the legend, to show results computed for different $\Omega_{0}$.

As first observation we find that our results are to a good approximation independent of the choice of bare phonon frequency. This is reflected in the fact that all three curves (different line styles) for any of the electron dispersions fall almost precisely on top of each other. Therefore we conclude that $\Omega_{\mathbf{q}} / \Omega_{0}$ is a direct function of $\lambda_{0}$ [see Fig. 4(a)] but not of $\Omega_{0}$. This can be seen by expressing the above functional form as $\Omega_{\mathbf{q}} / \Omega_{0}=\sqrt{1-\lambda_{0} \chi_{\mathbf{q}, l=0}^{0} / N_{0}}$ via Eq. (23). Notably, there is still an implicit dependence on $\Omega_{0}$ hidden in

$$
\chi_{\mathbf{q}, l}^{0}=-T \sum_{\mathbf{k}, m} \operatorname{Tr}\left[\hat{G}_{\mathbf{k}, m} \hat{\rho}_{3} \hat{G}_{\mathbf{k}+\mathbf{q}, m+l} \hat{\rho}_{3}\right]
$$

due to the self-consistency of our approach, which is why the curves are not precisely equivalent.

When comparing results computed for different electron fillings we find much bigger effects in the renormalized phonon spectrum. Starting with the orange curves of Fig. 4(b), we find the most pronounced phonon softening at $\mathbf{q}=(\pi, \pi)$. This case is already discussed above, and can be explained by well-satisfied nesting conditions at this wave vector. The green curves show larger values for $\Omega_{\mathbf{q}} / \Omega_{0}$, i.e., the renormalization effects are less pronounced compared to results shown in orange. From the FS properties [see Fig. 2(b)], we know that the exchange momentum is no longer close to $M$ when focusing on the green curve, rather $\mathbf{q}$ lies in between $\Gamma$ and $M$ or $X$. This nesting property is directly translated into results of Fig. 4(b), where the softest phonons are found along $\Gamma-M$ and $X-\Gamma$. Turning now to the lowest electron filling, the purple lines (3) in Figs. 4(b) and 2(a), the phonon frequencies $\Omega_{\mathbf{q}}$ are almost as large as their respective $\Omega_{0}$, for $\mathbf{q}$ between $M$ and $X$. Since the FS is a very small circle at the center of the BZ, it is not surprising that the susceptibility peaks only at small wave vectors. Therefore we observe softer phonons around $\Gamma$ in these results.

In the discussion above we did identify FS nesting as an important factor when considering phonon softening and renormalized couplings in two dimensions. We now want to explore this aspect also in 3D systems, using electron energies from Fig. 2(c). Fixing $\Omega=100 \mathrm{meV}$, we solve the Eliashberg equations for three different coupling strengths, $\lambda_{0}=0.05$, 0.1 , and 0.15 , at $T=20 \mathrm{~K}$. The corresponding results for the phonon spectrum are shown in Figs. 4(c)-4(e), respectively. Each of these panels contains results for all three electron fillings tested, where we adopt the color code of Figs. 2(c)-2(f). The first observation is similar to the 2D case, i.e., the phonons become softer as we increase the coupling strength. This can be seen by comparing similarly colored curves among different panels of Figs. 4(c)-4(e). Further it is apparent that the phonon softening is generally less pronounced and coherent in three dimensions. The orange curves (1) in each panel show a relatively small tendency for phonon softening, while the smallest $\Omega_{\mathbf{q}} / \Omega_{0}$ are detected at $M$ and $R$. Effects are even less prominent for the green lines (2) of each panel, where, for couplings up to $\lambda_{0}=0.15, \Omega_{\mathbf{q}} / \Omega_{0}$ stays relatively close to unity throughout the BZ. For the smallest electron filling (3), which is represented by purple lines in Figs. 4(c)-4(e), we find the results with highest anisotropy. Throughout most parts of the BZ the phonon spectrum is to a good approximation not renormalized, but $\Omega_{\mathbf{q}} / \Omega_{0}$ decreases strongly around $\Gamma$. This behavior is qualitatively similar to the $2 \mathrm{D}$ case shown in Fig. 4(b), in contrast to the other curves shown here (green, orange).

From the above discussion we learn that reduced nesting properties lead to less phonon softening in three dimensions, compared to the 2D situation. However, very small electron fillings are an exception to this trend because we observe comparable results of $\Omega_{\mathbf{q}} / \Omega_{0}$ in two and three dimensions. The reason lies in the special role of the $\Gamma$ point: When considering a spherical FS, $\mathbf{q} \sim(0,0,0)$ is the only exchange vector with which FS parts can be connected without dependence on the angle of wave vector $\mathbf{q}$, hence the susceptibility response in three dimensions can develop similarly coherent contributions at $\Gamma$ as in two dimensions.

We now turn to a more detailed discussion of the renormalized coupling strength $\lambda$, which, as we argue below, does similarly as the phonon softening strongly depend on FS nesting properties, and hence follows different trends in two and three dimensions. As stated before, the coupling diverges as we increase $\lambda_{0} \rightarrow \lambda_{0}^{\star}$, with $\lambda_{0}^{\star}$ marking a lattice instability. Recalling the definition $\lambda=\left\langle\left\langle\lambda_{\mathbf{k}-\mathbf{k}^{\prime}}\right\rangle_{\mathbf{k} \in \mathrm{FS}}\right\rangle_{\mathbf{k}^{\prime} \in \mathrm{FS}}$, we combine Eqs. (24) and (7) to write the momentum dependent coupling as

$$
\lambda_{\mathbf{q}}=N_{0} g_{0}^{2}\left[\frac{\Omega_{0}}{2}+\Pi_{\mathbf{q}, l=0}\right]^{-1},
$$

where we used $g_{\mathbf{q}}=g_{0}$. With $\Pi_{\mathbf{q}, l=0}=-g_{0}^{2} \chi_{\mathbf{q}, l=0}$ and $\lambda_{0}=$ $2 g_{0}^{2} N_{0} / \Omega_{0}$, we get

$$
\begin{aligned}
\lambda & =\left\langle\left\langle\lambda_{\mathbf{k}-\mathbf{k}^{\prime}}\right\rangle_{\mathbf{k} \in \mathrm{FS}}\right\rangle_{\mathbf{k}^{\prime} \in \mathrm{FS}} \\
& =\left\langle\left\langle\frac{\lambda_{0}}{1-\lambda_{0} \chi_{\mathbf{q}, l=0} / N_{0}}\right\rangle_{\mathbf{k} \in \mathrm{FS}}\right\rangle_{\mathbf{k}^{\prime} \in \mathrm{FS}} .
\end{aligned}
$$



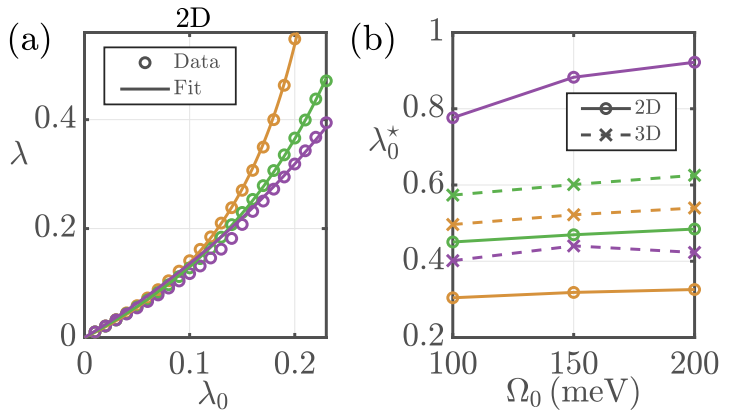

FIG. 5. (a) Renormalized coupling strength as function of $\lambda_{0}$, calculated in two dimensions for $\Omega_{0}=100 \mathrm{meV}$ and $T=20 \mathrm{~K}$. Open circles represent our self-consistent results; solid lines are obtained from Eq. (31). (b) Critical couplings $\lambda_{0}^{\star}$ as function of $\Omega_{0}$, shown for different dispersions in two and three dimensions. The color code used here in panels (a) and (b) reflects the choice of electron dispersion according to Figs. 2(a) and 2(c). Results obtained for two dimensions (three dimensions) are shown via solid (dashed) lines.

We model Eq. (30) by introducing the fitting function

$$
\lambda \sim a_{\lambda} \frac{\lambda_{0}}{1-b_{\lambda} \lambda_{0}},
$$

where $a_{\lambda}$ reflects the magnitude of the coupling, and $b_{\lambda}$ is a measure of the influence due to $\chi_{\mathbf{q}, l=0} / N_{0}$. Needless to say, Eq. (31) is an approximation, since we neglect the momentum dependence of the susceptibility. As mentioned earlier, the renormalized coupling diverges at a critical choice $\lambda_{0}^{\star}$ of the bare coupling strength. We can find an estimate of this quantity from the denominator in Eq. (31), namely, when $\left(1-b_{\lambda} \lambda_{0}\right) \rightarrow 0$ we can write

$$
\lambda_{0}^{\star} \simeq \frac{1}{b_{\lambda}}
$$

In Fig. 5(a) we show our self-consistent results for $\lambda$ as function of $\lambda_{0}$ as open circles, for $\Omega_{0}=100 \mathrm{meV}, T=20 \mathrm{~K}$, and the three 2D electron dispersions of Fig. 2(a) (similar color code). The fitted behavior as described in Eq. (31) is shown as solid lines for each data set. As is apparent from this graph, the renormalized coupling strength can be modeled with the above functional form to a very good approximation. Repeating the same procedure for $\Omega_{0}=150$ and $200 \mathrm{meV}$, and for all three 3D electron energies leads to the curves shown in Fig. 5(b), where we plot the critical bare coupling as function of input frequency. Solid (dashed) lines and open circles (crosses) represent trends in two dimensions (three dimensions), while colors are again corresponding to the electron dispersions in Figs. 2(a) and 2(c).

From all curves of Fig. 5(b) we learn that $\lambda_{0}^{\star}$ is to first-order approximation independent of the initial phonon frequency, which goes in line with observations from Fig. 4(b). Further, our 2D results reflect the expected behavior with respect to nesting conditions: The orange dispersion (1) of Fig. 2(a) is relatively well nested, therefore the susceptibility shows large contributions that are reflected in the fitting constant $b_{\lambda}$. Consequently, the critical input coupling [compare Eq. (32)] is a small number. As we proceed to the green (2), and eventually the purple (3) electron dispersion, the nesting becomes less
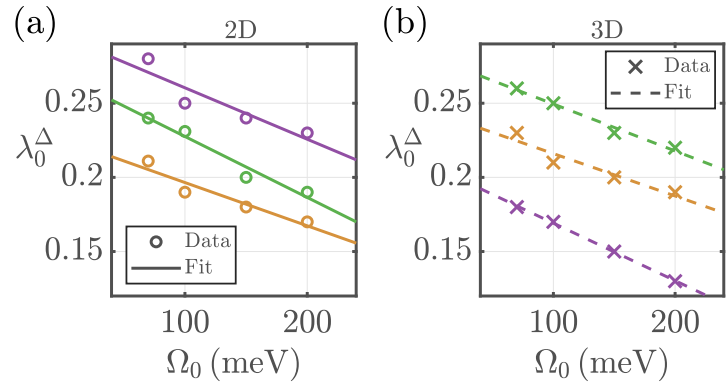

FIG. 6. Bare coupling strength $\lambda_{0}^{\Delta}$, corresponding to the onset of superconductivity, as function of input frequency $\Omega_{0}$. Results are computed for $T=20 \mathrm{~K}$, and the color code corresponds to Fig. 2 . (a) Results for $2 \mathrm{D}$ electron dispersions. (b) Results for 3D electron dispersions.

coherent, so that the resulting $\lambda_{0}^{\star}$ grows larger. Turning to the dashed curves of Fig. 5(b) we find a different behavior. The values for $\lambda_{0}^{\star}$ are far less susceptible for changes in the electron filling. Further, we find smaller values for $b_{\lambda}$ in three dimensions than in two dimensions, which clarifies that nesting in three dimensions is less important. An exception to this is the result drawn in purple, representing the most shallow electron dispersion (3). As discussed in connection to Figs. 4(c)-4(e), this stems from the special role of nesting at $\mathbf{q} \sim(0,0,0)$, which is developed to a larger extent in three dimensions than in two dimensions. For this reason (large value of $b_{\lambda}$ ) the purple dashed line not only falls below the two other curves for 3D systems, but lies also lower than the purple solid line obtained for the most shallow 2D electron dispersion (3).

Note that the results of Fig. 5(b) are obtained by fitting $\lambda$ in the noncritical range of the input coupling, i.e., for $\lambda_{0}$ significantly smaller than $\lambda_{0}^{\star}$. Therefore the reported values of $\lambda_{0}^{\star}$ are to be interpreted as qualitative trends, and should not be taken as precise numbers. Performing a calculation at $\lambda_{0} \sim \lambda_{0}^{\star}$ is numerically very difficult, because the input $\xi_{\mathbf{k}}-\delta \mu$ has to be gradually adjusted so as to keep the electron filling at the desired level. If, however, the interplay of $\delta \mu, \Omega_{0}$, and $\lambda_{0}$ is such that the input coupling is above the respective $\lambda_{0}^{\star}$, the self-consistent Eliashberg loop never converges.

We end this section by looking into the bare coupling strength $\lambda_{0}^{\Delta}$, at which the onset of superconductivity occurs, i.e., the smallest $\lambda_{0}$ at which $\Delta_{\mathbf{k}, m} \neq 0$. As is apparent from Fig. $3, \lambda_{0}^{\Delta}$ depends both on properties of the electron dispersion and on the phonon frequency, hence we want to examine this quantity closer in two and three dimensions. We choose phonon frequencies $\Omega_{0}$ as $70,100,150$, or $200 \mathrm{meV}, T=$ $20 \mathrm{~K}$ and use electron energies from Figs. 2(a) and 2(c) to show computed results for $\lambda_{0}^{\Delta}$ as function of $\Omega_{0}$ in Figs. 6(a) and 6(b), for two and three dimensions, respectively. Open circles (crosses) represent our data, while solid (dashed) lines are obtained by a linear fit of the 2D (3D) data. The color code corresponds to choices of electron dispersion according to Fig. 2. For each of the curves, both in two and three dimensions, we observe a linear decrease in $\lambda_{0}^{\Delta}$ with increasing frequency. This stems from the fact that renormalized frequencies $\Omega_{\mathbf{q}}$ are growing with $\Omega_{0}$, enhancing the tendencies of the system to form a superconducting state. 

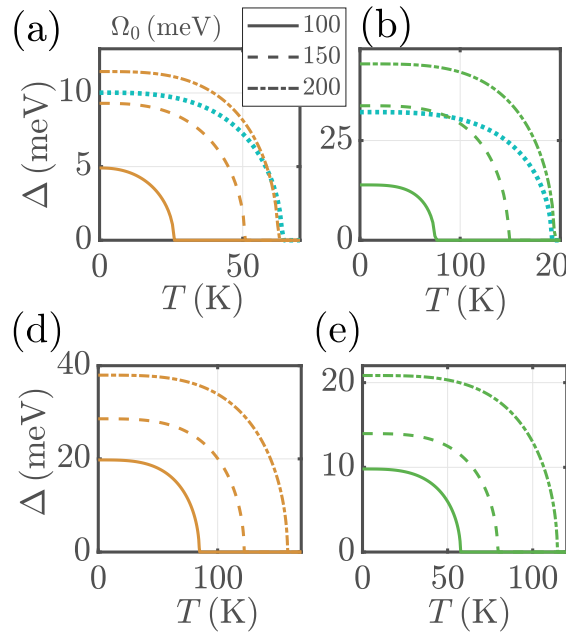

(e)
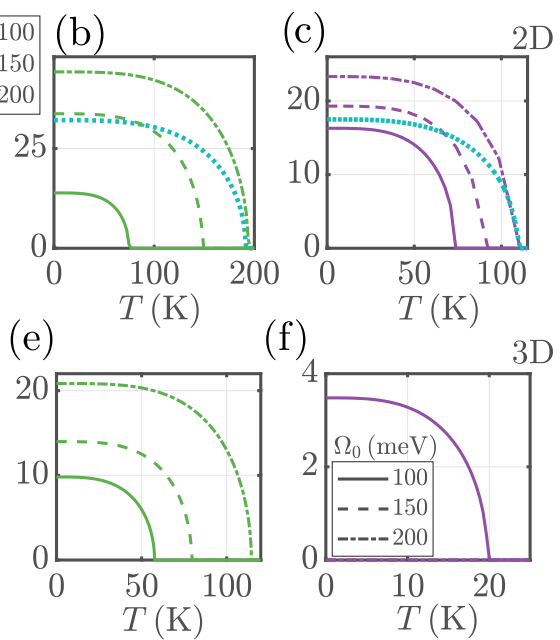

(f)

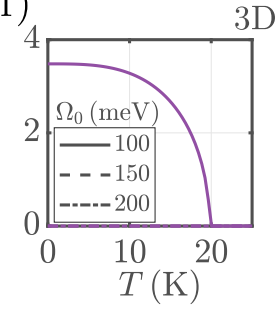

FIG. 7. Maximum superconducting gap as function of temperature with color code corresponding to the electron dispersions of Figs. 2(a) and 2(c). The different line styles represent the choices of initial frequency $\Omega_{0}$ as written in the legends. For the size of input couplings $\lambda_{0}$ see main text. (a-c) Results for $\Delta(T)$ for the 2D electron systems. Each panel contains an example for $\Delta(T)$ shown by the cyan dotted curve, that is obtained by setting $\Pi_{\mathbf{q}, l}=0$, $\Omega_{0}=200 \mathrm{meV}$, and matching $T_{c}$ to the corresponding dot-dashed curve. (d-f) Results for the 3D systems of Fig. 2(c), with coupling strengths as described in the main text.

When comparing results for $\lambda_{0}^{\Delta}$ with respect to shallowness and nesting, we find similar trends with those already observed in Figs. 4 and 5. In the 2D case [Fig. 6(a)], the orange dispersion (1) has the best nesting conditions, resulting in a large susceptibility at the nesting wave vector. This in turn leads to a relatively large renormalized coupling strength $\lambda$. By increasing shallowness of the electron energies, hence considering the green (2) and purple (3) lines, we obtain decreasing magnitudes of $\chi_{\mathbf{q}, l}$, which results in smaller coupling strengths. Hence, to achieve similar values of $\lambda$ as in the well nested case, $\lambda_{0}$ has to be increased. For $3 \mathrm{D}$ systems nesting is generally less coherent, therefore the orange (1) and green (2) curves in Fig. 6(b) show larger values for $\lambda_{0}^{\Delta}$ than in Fig. 6(a), with similar reasoning. The very shallow $\xi_{\mathbf{k}}(3)$, represented by the purple line, is again the exception due to exhibiting coherent nesting behavior at $(0,0,0)$, which is why only small couplings are needed to induce superconductivity.

\section{CRITICAL TEMPERATURES}

Let us now turn to the superconducting critical temperature $T_{c}$ that can be determined by following the maximum superconducting gap with $T$. We define $T_{c}$ as the smallest temperature at which $\Delta$ vanishes and use the same electron dispersions as before [see Figs. 2(a) and 2(c)]. Starting in two dimensions, we test the initial frequencies $\Omega_{0}$ being 100 , 150 , or $200 \mathrm{meV}$, which are represented in Figs. 7(a)-7(c) by solid, dashed, and dot-dashed lines, respectively. All panels show the maximum superconducting gap as function of temperature, with color code corresponding to the choice of electron dispersion from Fig. 2(a). For each $\xi_{\mathbf{k}}$ and $\Omega_{0}$ we take the largest value of $\lambda_{0}<\lambda_{0}^{\star}$ for which a solution could be stabilized, so as to maximize the renormalized coupling and critical temperature. Furthermore, each panel contains the result for calculating $\Delta(T)$ without finite phonon self-energy, i.e., setting $\Pi_{\mathbf{q}, l}=0$, shown by the dotted cyan curves. For each electron filling we compute these curves by setting $\Omega_{0}=$ $200 \mathrm{meV}$ and matching $T_{c}$ with the respective dot-dashed result.

For Fig. 7(a) we take the parameter choices $\left(\Omega_{0}, \lambda_{0}\right)=$ (100 meV, 0.205), (150 meV, 0.215), and (200 meV, 0.22). The resulting critical temperatures lie between 26 and $65 \mathrm{~K}$. Next we turn to Fig. 7(b): The Fermi surface is smaller than in the case before, but the electron band (2) is not yet very shallow. We use $\left(\Omega_{0}, \lambda_{0}\right)=(100 \mathrm{meV}, 0.34)$, (150 meV, 0.41), and (200 meV, 0.42) for the solid, dashed, and dot-dashed curves. As easily observed, $T_{c}$ drastically grows when compared to Fig. 7(a), with the largest critical temperature almost reaching $200 \mathrm{~K}$. When we finally go to the results for a shallow band (3) [Fig. 7(c)], $T_{c}$ ranges between $\approx 80$ and $\approx 110 \mathrm{~K}$. Here the curves are produced by choosing $\left(\Omega_{0}, \lambda_{0}\right)=(100 \mathrm{meV}, 0.54),(150 \mathrm{meV}, 0.53)$, and (200 meV, 0.55).

The here observed results can be explained by an intuitive picture: The dispersion corresponding to Fig. 7(a) exhibits the best nesting conditions, which leads to enhanced renormalization of the phonon propagator (see also Sec. IV). This means that a substantial part of the available coupling in the system is "used" for renormalizing the phonon frequency, leaving less coupling available for Cooper pairing and therefore resulting in a reduction of $\Delta$ and $T_{c}$. In the opposite limit of the shallow band (3) [Fig. 7(c)], most of the initial coupling strength is available to form the superconducting state, because the nesting, and therefore the renormalization of the phonon spectrum, is comparatively minor. Consequently $T_{c}$ is enhanced when comparing to Fig. 7(a). However, due to the shallowness of the electron band (3) we face a reduced FS area, therefore the $T_{c}$ 's are larger than in Fig. 7(a), but not maximized. When comparing the results for $\Omega_{0}=200 \mathrm{meV}$ to the cyan dotted curve, found for $\Pi_{\mathbf{q}, l}=0$, in each panel we observe larger values for $\Delta / k_{\mathrm{B}} T_{c}$ when the phonon self-energy is finite. This comparison points towards stronger coupling when including the self-consistent renormalization of the phonon spectrum, which is rather intuitive when considering that the maximally possible $\lambda_{0}<\lambda_{0}^{\star}$ has been chosen here, leading to comparatively large values for the renormalized electronphonon coupling strength $\lambda$.

The results shown in Fig. 7(b) can be seen as example of how to achieve the highest possible critical temperatures. The electron dispersion (2) exhibits relatively bad nesting conditions, while still not being in the limit of a very shallow band. For achieving a large magnitude of $\Delta$ (and therefore $T_{c}$ ) a substantial renormalized coupling $\lambda$ is required, combined with a large FS. The system of Fig. 7(b) lies in an ideal intermediate regime, where the balance between phonon renormalization and electron band shallowness is kept. The FS nesting condition is good enough for achieving a large $\lambda$, but not as ideal as in Fig. 7(a) so as to drive the system towards a lattice instability before superconductivity can build up. Additionally the FS size is sufficiently large, in contrast to Fig. 7(c), so as to boost values for $T_{c}$. 
The above interpretation is the intuitive extension of results well known for the approximation $\Pi_{\mathbf{q}, l}=0$. If only the bare phonon propagator is considered, one recovers the standard BCS result, i.e., the superconducting critical temperature depends on the phonon frequency and the FS size. An increase in either of those quantities leads to a growing $T_{c}$. In contrast, as soon as $\Pi_{\mathbf{q}, l}$ is finite, the values of the maximum superconducting gap and critical temperature decrease to an extent that depends only on the nesting conditions [compare Figs. 7(a)7(c)]. Therefore there should exist an ideal balance between phonon renormalization and FS size, which maximizes the critical temperature.

Let us now turn to 3D systems, taking electron energies $\xi_{\mathbf{k}}$ from Fig. 2(c). The maximum superconducting gap as function of temperature is shown in Figs. 7(d)-7(f), where we test again three phonon frequencies for each electron filling (see legend). Each curve is obtained by imposing a respective input coupling $\lambda_{0}$ that is close to the lattice instability. In Fig. 7 (d) we plot our self-consistent solutions for $\left(\Omega_{0}, \lambda_{0}\right)=$ (100 meV, 0.383), (150 meV, 0.4), and (200 meV, 0.42), using the orange electron dispersion (1) of Fig. 2(c). As before we find a clear enhancement in the maximally allowed $\Delta$ and $T_{c}$ with increased phonon frequency. Results for both maximum superconducting gap and critical temperature become comparatively smaller in Fig. $7(\mathrm{e}),\left(\Omega_{0}, \lambda_{0}\right)=$ (100 meV, 0.31), (150 meV, 0.31), (200 meV, 0.33), where the green dispersion of Fig. 2(c) is used. The reason for this trend is a decrease in FS size, combined with a less important role of nesting properties in 3D systems. Finally, we show $\Delta(T)$ for the purple 3D dispersion (3) [see Fig. 2(c)], in Fig. 7(f). Here we found no superconductivity for $\left(\Omega_{0}, \lambda_{0}\right)=$ $(200 \mathrm{meV}, 0.15)$ and $(150 \mathrm{meV}, 0.12)$ down to $10 \mathrm{~K}$. The gap size and $T_{c}$ for $\left(\Omega_{0}, \lambda_{0}\right)=(100 \mathrm{meV}, 0.1)$ are substantially smaller than in Figs. 7(d) and 7(f). These results can be explained under the light of an extremely small FS of the purple electron dispersion (3). Additionally, large parts of the available coupling are used for renormalizing the phonon spectrum, due to well-enhanced nesting conditions at $\mathbf{q} \sim$ $(0,0,0)$; see also discussions in Sec. IV.

On a related note, our temperature-dependent results enable us to explicitly test the well-established practice of using the same phonon frequencies for all temperatures across the superconducting phase transition. The phonon spectra are usually calculated in the normal state, i.e., without taking Cooper pairing into account, and afterwards used for describing the superconducting pair condensate at $T<T_{c}$. When considering $\Omega_{\mathbf{q}}$ as function of $T$, we find that this is indeed a valid approximation. For the model calculations done here, the changes are in the sub-meV range, with $\Omega_{\mathbf{q}}$ slightly growing as $T$ increases. This rather insignificant trend can be attributed to thermal broadening, and can conveniently be neglected to very good approximation.

Above we have already encountered some aspects concerning the superconducting $T_{c}$, in both $2 \mathrm{D}$ and $3 \mathrm{D}$ systems. In the following we propose a scaling of the critical temperature, which can also serve as approximate upper bound. In Fig. 8 we show our maximally possible values for $T_{c}$ as function of renormalized phonon frequency, adopting the color code from Fig. 2. Our computed results for 2D and 3D systems are, respectively, shown in Figs. 8(a) (open circles) and 8(b)
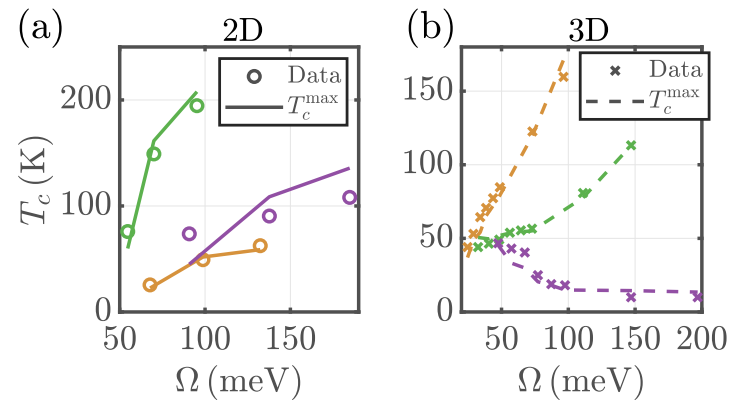

FIG. 8. Influence of phonon renormalization on $T_{c}$ in 2D and 3D systems. Open circles and crosses represent computed values for $T_{c}$ as function of renormalized frequency $\Omega$, where the color code of Fig. 2(a) (2D) and Fig. 2(c) (3D) is used for panels (a) and (b). The couplings have been chosen close to the lattice instability. In the main text we describe how to obtain the approximate upper bounds for $T_{c}$ as shown by the dashed lines.

(crosses). For finding these critical temperatures we choose initial frequencies $\Omega_{0} \in[50,200] \mathrm{meV}$, and pick the largest $\lambda_{0}<\lambda_{0}^{\star}$ for which a solution can be stabilized.

Our results show that the maximum $T_{c}$ obtained here always increases as function of renormalized frequency $\Omega=$ $\left\langle\Omega_{\mathbf{q}}\right\rangle_{\mathbf{q} \in \mathrm{BZ}}$ for $2 \mathrm{D}$ systems. However, in three spatial dimensions this trend is not observed for our most shallow dispersion (3), where $T_{c}<10 \mathrm{~K}$ for $\Omega \gtrsim 150 \mathrm{meV}$. For modeling the functional behavior found in Fig. 8, it is worthwhile considering trends reported by other authors. In Ref. [9], Esterlis et al. proposed that $T_{c}^{\max } \propto \Omega$ constitutes a reasonable upper bound for the critical temperature. However, our results do not appear linear in the renormalized phonon frequency, which is why this scaling might be a too crude approximation. Another proposal was made by Moussa and Cohen, stating that $T_{c}^{\max } \propto \sqrt{\Omega_{0}^{2}-\Omega^{2}}$ [37]. Imposing this form does indeed lead to satisfying agreement with our data in two dimensions, but fails to capture the decrease in critical temperature observed for the purple line in Fig. 8(b). We find that the best fit to our numerical data is given by the scaling expression

$$
k_{B} T_{c}^{\max } \propto \lambda(\Omega) \sqrt{\Omega_{0}^{2}-\Omega^{2}},
$$

which is shown as dashed lines in Fig. 8. As is apparent from comparing our data with outcomes of Eq. (33), the functional dependence in Fig. 8 is quite accurately captured. Especially for 3D systems the inclusion of $\lambda(\Omega)$ is crucial to mimic the observed trends, while the functional dependence proposed in Ref. [37] would suffice for the 2D cases.

It is important to notice the difference between Eq. (33) and the more simplified scaling law of Ref. [37], since $\lambda$ is not constant with respect to $\Omega$. To show this explicitly, we can solve Eq. (25) for the zero-frequency phonon self-energy, yielding $\Pi_{\mathbf{q}, l=0}=\left(\Omega_{\mathbf{q}}^{2}-\Omega_{0}^{2}\right) /\left(2 \Omega_{0}\right)$. We insert this expression into Eq. (29) and, as an approximation, replace $\Omega_{\mathbf{q}}$ by $\Omega$, so that

$$
k_{B} T_{c}^{\max } \sim \lambda_{0} \frac{\Omega_{0}^{2}}{\Omega^{2}} \sqrt{\Omega_{0}^{2}-\Omega^{2}} .
$$

Here it is furthermore worthwhile to note the functional dependence of the renormalized phonon frequency and bare 
coupling strength within our model, i.e., $\lambda_{0}=\lambda_{0}\left(\xi_{\mathbf{k}}, \Omega_{0}\right)$ and $\Omega=\Omega\left(\xi_{\mathbf{k}}, \Omega_{0}\right)$.

The interpretation of Eqs. (33) and (34) is rather intuitive: The highest possible value of $T_{c}$ depends directly on the renormalized coupling strength in the system. Additionally, well-developed phonon softening is advantageous for maximizing the critical temperature, i.e., having $\Omega \ll \Omega_{0}$. These two effects are not decoupled from each other, since both depend on nesting properties and the FS size. For two dimensions we already observed the trends in Figs. 7(a)-7(c); the green curve in Fig. 8(a) represents the ideal compromise between magnitude of the FS (purple curve being suppressed because the FS is too small) and nesting properties (orange curve being suppressed because phonon softening is too strong). Going from the orange to the green curve in Fig. 8(b) reflects the decrease of FS size (compare Fig. 2). The most shallow 3D system exhibits additionally coherent nesting at $\Gamma$, so as to produce negative phonon frequencies before superconductivity can fully develop. Summarizing, we obtain numerically that the effect of phonon softening is in general favorable for superconductivity, provided that the unrenormalized phonon frequency $\Omega_{0}$ is sufficiently large, such that the system is not developing a lattice instability before superconductivity can fully build up.

\section{CONCLUSIONS}

In this paper we have investigated the details of Eliashberg theory including self-consistent phonon renormalization on a model basis. We worked out the similarities and differences between 2D and 3D systems, identifying nesting properties and FS size as key aspects. From those follow directly the trends for phonon softening, electron-phonon renormalization, and the critical temperature. Our paper therefore constitutes an extensive overview of possible effects of phonon renormalization in Eliashberg theory under Migdal's approximation.

Our calculations show that the maximally possible $T_{c}$ in two dimensions results from a delicate interplay between FS size and nesting properties. If the size of the FS is too small, no strongly increased values for $T_{c}$ are found. On the other hand, very coherent nesting, which in our model is associated with a big FS, drives the system too quickly towards a lattice instability with increasing coupling strength. Maximal values for the critical temperature are therefore found in an intermediate regime. Comparable values for $T_{c}$ when neglecting the phonon renormalization are found for less strongly coupled systems. In three spatial dimensions, nesting, and therefore the renormalization of the phonon spectrum, becomes less coherent. Consistently we find that the magnitude of $T_{c}$ is mainly dictated by the size of the FS.

In Sec. IV we have shown that there exists an inherent bound $\lambda_{0}^{\star}$ on the maximal coupling strength, which is not to be confused with critical values of $\lambda_{0}$ in other works. Chubukov et al. use the notation $\lambda_{\text {cr }}$ to describe the border of applicability of Eliashberg theory with respect to the electron-phonon coupling strength [7]. This border is found by comparing outcomes from Eliashberg theory with QMC simulations in the normal state, so that for $\lambda>\lambda_{\text {cr }}$ the two theories no longer produce similar results. The same border of applicability is denoted $\lambda^{\star}$ in Ref. [8], which, again is not the same coupling strength as our $\lambda_{0}^{\star}$ corresponding to a lattice instability.

As mentioned before, various authors agree on the existence of a maximal coupling strength up to which Eliashberg theory produces accurate outcomes. However, this aspect seems not to be understood completely yet. It has been claimed that Migdal-Eliashberg theory breaks down at an input coupling $\lambda_{0} \simeq 0.4$, which was concluded by considering a $2 \mathrm{D}$ Holstein model $[7,8]$. However, it is by no means clear whether the same limit exists in real materials, and in particular in 3D systems, as was implied, e.g., in Ref. [9]. Furthermore, other authors have arrived at deviating conclusions: In an early work by Marsiglio [4] good agreement between QMC and Eliashberg theory was found regardless of coupling strength, provided that the phonon renormalization is taken self-consistently into account. We therefore conclude that the correct picture is currently still elusive, and want to stress again that results presented here are solely derived within Migdal's approximation.

There are several aspects that go beyond the scope of the current paper, but are important to be considered in future studies for gaining a better understanding of the formalism. Examples of such extensions would be a nontrivial momentum dependence in the electron-phonon coupling $\lambda_{\mathbf{q}}$, or replacing the Einstein phonon frequency $\Omega_{0}$ by a wave vector and phonon branch $v$ dependent frequency $\omega_{\mathbf{q}, v}$. Especially a $v$ dependence would require a slight generalization of the equations used here. For simplicity, we have neglected any Coulomb repulsion, which should be included in our treatment as well, ideally in a more exploratory manner than just following the most commonly used practice of setting $\mu^{\star}=0.1$ [6]. Another major step further would be to extend the vertex-corrected Eliashberg theory of Ref. [27] by a selfconsistent phonon renormalization, which would then have to be done by including up to the second-order processes in both electron and phonon self-energies.

Here we have solely considered optical phonon modes but it is worthwhile noting that potentially deviating results are to be expected for acoustic modes (characterized by $\left.\omega_{\mathbf{q}=(0,0), v}=0\right)$. We have seen that the momentum dependent decrease in the magnitudes of optical phonon frequencies generally follows the nesting wave vector. However, for acoustic frequencies this is not necessarily true, since the corresponding electron-phonon interaction $\lambda_{\mathbf{q}, v}$ must then acquire a nontrivial momentum dependence, which has the constraint $\lambda_{\mathbf{q}=(0,0), v}=0$. Further, if a multibranch system is considered, not all branches are expected to be softened to the same extent, which makes it rather difficult to predict the outcome of calculations similar to those we performed here for our model systems.

Additionally, we note that real materials have a degree of freedom not incorporated in our theory, which is structural transformations. A good example for discussing this phenomenon is the high-temperature superconducting hydrides $[38,39]$. These materials undergo one or more structural phase transitions when subject to external pressure (see, e.g., Ref. [40]). By calculating phonon spectra via density functional theory it is found that phonon softening is responsible for such structural reconfigurations, since nonstable phases lead to imaginary frequencies at the nesting wave vector. 
Therefore, for either optical or acoustic phonon modes, the actual system adopts an atomic configuration that is stable with respect to the lattice vibrations. Our analysis is valid for testing different coupling strengths within one stable phase, but not across such phase transitions.

\section{ACKNOWLEDGMENTS}

This work has been supported by the Swedish Research Council, the Röntgen-Ångström Cluster, the Knut and Alice Wallenberg Foundation (Grant No. 2015.0060), and the Swedish National Infrastructure for Computing (SNIC).

\section{APPENDIX: BENCHMARK CALCULATIONS}

In the following we benchmark our formalism by comparing it to existing literature to show that our implementation is reliable and in agreement with other approaches. Specifically, we compare our results with those of Ref. [8], where the authors compare outcomes of quantum Monte Carlo calculations with results from Migdal-Eliashberg theory, computing the charge density wave and superconducting susceptibilities. The temperatures considered in this work exceed the phase transition, i.e., the system is in the interacting but nonsuperconducting state. For their Eliashberg calculations the authors include the electron and phonon lowest-order Feynman diagrams, similar to our present paper. The aim in this section is to reproduce Figs. 3 and 4 published in Ref. [8].

Esterlis et al. [8] use a two-dimensional tight-binding model with nearest- and next-nearest-neighbor hopping energies $t$ and $t^{\prime}$. Their ratio is taken as $t^{\prime} / t=-0.3$. The electron filling is fixed at $n=0.8$. For both figures that we are interested in, the temperature is $T=t / 16$, the adiabaticity ratio is $\Omega_{0} / E_{F}=0.1$ (with $E_{F}$ the Fermi energy), and the coupling strength is chosen from $\lambda_{0} \in\{0.2,0.4,0.5\}$. The functional form of their tight-binding approach reads

$$
\xi_{\mathbf{k}}=-2 t\left[\cos \left(k_{x}\right)+\cos \left(k_{y}\right)\right]-4 t^{\prime} \cos \left(k_{x}\right) \cos \left(k_{y}\right)-\mu
$$

for the electron energies [41]. The chemical potential $\mu$ has to be adjusted so as to fix the electron filling at the chosen value. In contrast to our theory presented here, Esterlis et al. [8] have focused on properties above $T_{c}$ using a scalar function $\Sigma\left(\mathbf{k}, \omega_{m}\right)$, while our Nambu formalism allows us to directly access superconducting properties. We can make contact between the two formulations by considering only the (11) matrix element of $\hat{\Sigma}_{\mathbf{k}, m}$ :

$$
-\operatorname{Im} \hat{\Sigma}_{\mathbf{k}, m}^{(11)}=\omega_{m}\left(Z_{\mathbf{k}, m}-1\right),
$$

which is equivalent to the scalar self-energy of Ref. [8].

In Figs. 9(a)-9(c) we show our results for Eq. (A2) as solid lines in all three panels, corresponding to different choices of $\lambda_{0}$. We plot $-\operatorname{Im} \hat{\Sigma}_{\mathbf{k}, m}^{(11)}$ at those FS momenta, that produce the maximal (blue curve) and minimal (red curve) magnitude of the result. As comparison we extracted results from Ref. [8] at two different FS angles (see the orange and green dashed lines). It is directly apparent that the curves coincide to a very good degree. Next, we attempt to reproduce the renormalized
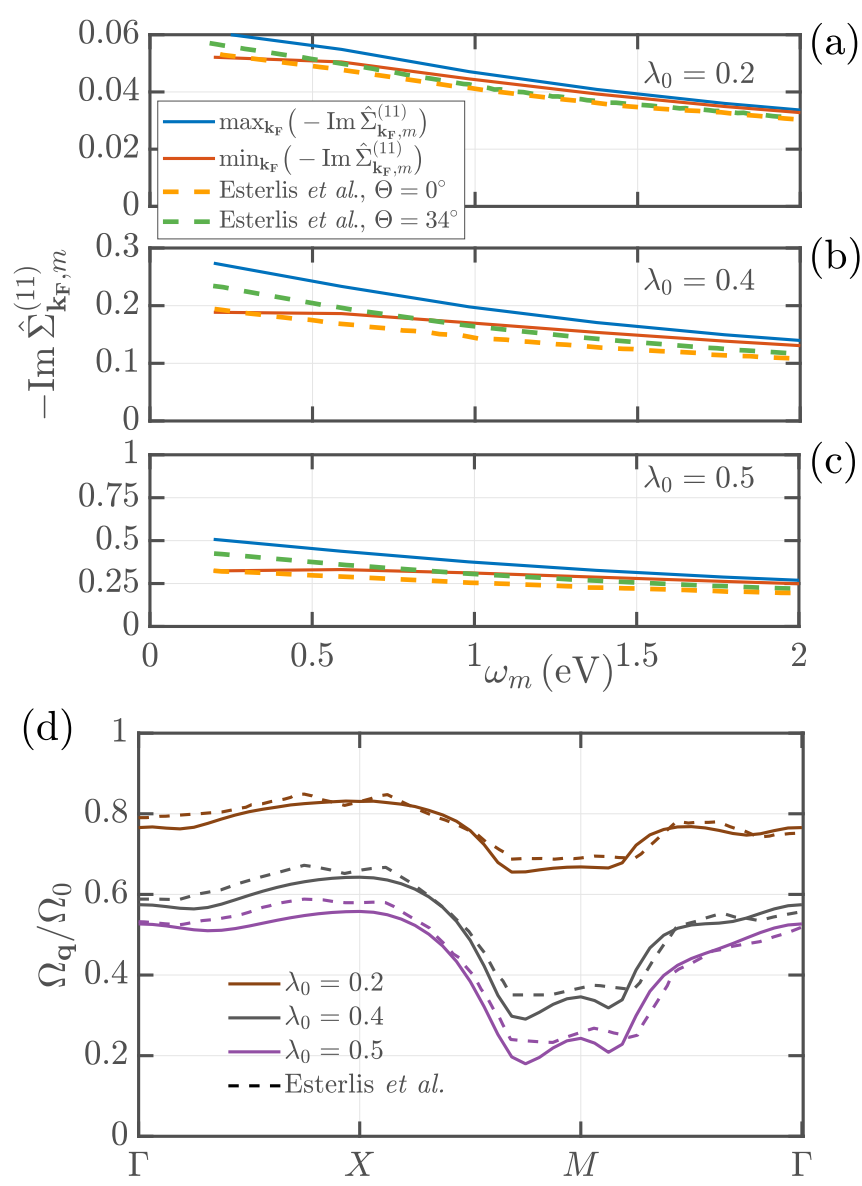

FIG. 9. (a-c) Imaginary part of the (11) element of the electron self-energy as function of Matsubara frequencies. Results shown in panels (a), (b), and (c) are obtained for $\lambda_{0}=0.2,0.4$, and 0.5 , respectively. Dashed curves are taken from Ref. [8], at FS angles as written in the legend. Blue and red solid lines correspond to our results computed from Eq. (A2), where we show curves at those FS momenta that produce the maximum (blue) and minimum (red) magnitude for the self-energy. (d) Phonon frequencies, normalized to the bare input $\Omega_{0}$, along high-symmetry lines of the BZ. Different colors represent input couplings as written in the legend. For each $\lambda_{0}$ we show our results as solid lines, and curves taken from Ref. [8] are shown with dashed lines.

phonon frequency spectrum of Ref. [8], which can be obtained with the same set of parameters as described above. We show our results for $\lambda_{0}=0.2,0.4$, and 0.5 in Fig. 9(d) via solid brown, gray, and purple lines, respectively. As before, we find good agreement with results by Esterlis et al., plotted as dashed curves in similar color code.

The small deviations between our results and those of Ref. [8] observed in Fig. 9 can be likely attributed to different choices for the momentum grid size and number of Matsubara frequencies. Further, potential differences can arise from numerical details of the implementation, e.g., the way of performing the momentum and energy integrals, or how the aspect of electron filling is handled. 
[1] G. M. Eliashberg, Sov. Phys. JETP 11, 696 (1960).

[2] J. Bardeen, L. N. Cooper, and J. R. Schrieffer, Phys. Rev. 108, 1175 (1957).

[3] A. B. Migdal, Sov. Phys. JETP 34, 996 (1958).

[4] F. Marsiglio, Phys. Rev. B 42, 2416 (1990).

[5] R. T. Scalettar, N. E. Bickers, and D. J. Scalapino, Phys. Rev. B 40, 197 (1989).

[6] F. Marsiglio, Ann. Phys. (NY) 417, 168102 (2020).

[7] A. V. Chubukov, A. Abanov, I. Esterlis, and S. A. Kivelson, Ann. Phys. (NY) 417, 168190 (2020).

[8] I. Esterlis, B. Nosarzewski, E. W. Huang, B. Moritz, T. P. Devereaux, D. J. Scalapino, and S. A. Kivelson, Phys. Rev. B 97, 140501(R) (2018).

[9] I. Esterlis, S. A. Kivelson, and D. J. Scalapino, npj Quantum Mater. 3, 59 (2018).

[10] J. Bauer, J. E. Han, and O. Gunnarsson, Phys. Rev. B 84, 184531 (2011).

[11] A. S. Alexandrov, Europhys. Lett. 56, 92 (2001).

[12] D. Meyer, A. C. Hewson, and R. Bulla, Phys. Rev. Lett. 89, 196401 (2002).

[13] C. A. Perroni, V. Cataudella, G. De Filippis, and V. M. Ramaglia, Phys. Rev. B 71, 054301 (2005).

[14] N. C. Costa, T. Blommel, W.-T. Chiu, G. Batrouni, and R. T. Scalettar, Phys. Rev. Lett. 120, 187003 (2018).

[15] P. M. Dee, K. Nakatsukasa, Y. Wang, and S. Johnston, Phys. Rev. B 99, 024514 (2019).

[16] P. M. Dee, J. Coulter, K. G. Kleiner, and S. Johnston, Commun. Phys. 3, 145 (2020).

[17] V. N. Kostur and B. Mitrović, Phys. Rev. B 48, 16388 (1993).

[18] O. Gunnarsson, V. Meden, and K. Schönhammer, Phys. Rev. B 50, 10462 (1994).

[19] E. J. Nicol and J. K. Freericks, Physica C 235-240, 2379 (1994).

[20] P. Miller, J. K. Freericks, and E. J. Nicol, Phys. Rev. B 58, 14498 (1998).

[21] J. P. Hague, J. Phys.: Condens. Matter 15, 2535 (2003).

[22] L. Pietronero and S. Strässler, Europhys. Lett. 18, 627 (1992).

[23] P. Benedetti, C. Grimaldi, L. Pietronero, and G. Varelogiannis, Europhys. Lett. 28, 351 (1994).
[24] P. Paci, E. Cappelluti, C. Grimaldi, and L. Pietronero, Phys. Rev. B 65, 012512 (2001).

[25] L. Boeri, E. Cappelluti, C. Grimaldi, and L. Pietronero, Phys. Rev. B 68, 214514 (2003).

[26] E. Cappelluti, S. Ciuchi, C. Grimaldi, and L. Pietronero, Phys. Rev. B 68, 174509 (2003).

[27] F. Schrodi, P. M. Oppeneer, and A. Aperis, Phys. Rev. B 102 024503 (2020).

[28] The UppSC code provides a package to self-consistently solve the anisotropic, multiband, and full-bandwidth Eliashberg equations for frequency-even and odd superconductivity mediated by phonons, charge fluctuations, or spin fluctuations on the basis of $a b$ initio calculated input.

[29] A. Aperis, P. Maldonado, and P. M. Oppeneer, Phys. Rev. B 92, 054516 (2015).

[30] J. Bekaert, A. Aperis, B. Partoens, P. M. Oppeneer, and M. V. Milošević, Phys. Rev. B 97, 014503 (2018).

[31] F. Schrodi, A. Aperis, and P. M. Oppeneer, Phys. Rev. B 99, 184508 (2019).

[32] F. Schrodi, A. Aperis, and P. M. Oppeneer, Phys. Rev. B 102, 014502 (2020).

[33] F. Schrodi, A. Aperis, and P. M. Oppeneer, Phys. Rev. B 102, 180501(R) (2020).

[34] B. Nosarzewski, M. Schueler, and T. P. Devereaux, Phys. Rev. B 103, 024520 (2021).

[35] F. Schrodi, A. Aperis, and P. M. Oppeneer, Phys. Rev. B 98, 094509 (2018).

[36] P. B. Allen and B. Mitrović, in Solid State Physics, Advances in Research and Applications, Vol. 37, edited by H. Ehrenreich, F. Seitz, and D. Turnbull (Academic, New York, 1983), pp. 1-92.

[37] J. E. Moussa and M. L. Cohen, Phys. Rev. B 74, 094520 (2006).

[38] C. J. Pickard, I. Errea, and M. I. Eremets, Annu. Rev. Condens. Matter Phys. 11, 57 (2020).

[39] J. A. Flores-Livas, L. Boeri, A. Sanna, G. Profeta, R. Arita, and M. Eremets, Phys. Rep. 856, 1 (2020).

[40] S. Azadi, B. Monserrat, W. M. C. Foulkes, and R. J. Needs, Phys. Rev. Lett. 112, 165501 (2014).

[41] M. Vekić, R. M. Noack, and S. R. White, Phys. Rev. B 46, 271 (1992). 